were no cough (4\%), minimal (7\%), mild (12\%), moderate $(34 \%)$, severe (35\%) and very severe (8\%). Participants reported median (IQR) cough severity VAS 60 (32-80) mm, LCQ 11.2 (8.6-14.4) and PGI-S 3 (moderate) (3-4). PGI-S was associated with cough severity VAS and LCQ (rho $=0.81$, $\mathrm{p}<0.001 ;$ rho $=-0.76, \mathrm{p}<0.001$ respectively). Cough severity VAS (median, IQR) was significantly different between PGI-S categories; no cough: 0 (0-5) mm, minimal: 11 (7-24) $\mathrm{mm}$, mild: $30(19-39) \mathrm{mm}$, moderate: 50 (39-61) mm, severe: 76 $(69-82) \mathrm{mm}$ and very severe: $91 \quad(83-100) \mathrm{mm} \quad(\mathrm{p}<0.001)$ (figure 1a). LCQ (median, IQR) was also significantly different between PGI-S categories; no cough: 20.3 (19.5-20.7), minimal: 17.0 (16.3-18.1), mild: 15.4 (14.0-17.3), moderate: 11.9 (10.1-13.9), severe: $8.7(7.3-10.4)$ and very severe: 6.8 (5.3$8.9)(\mathrm{p}<0.001)$ (figure $1 \mathrm{~b})$.

Conclusion The PGI-S scale is a simple tool that characterises cough severity in a format familiar to clinicians, and allows comparisons with other conditions. The PGI-S has a strong relation with validated cough measures such as VAS and LCQ. Future studies should investigate the reproducibility and clinically important threshold for change of the PGI-S.

\section{P58 THE PREVALENCE OF CHRONIC COUGH AMONGST FEMALES WITH STRESS URINARY INCONTINENCE}

${ }^{1} \mathrm{P}$ Cho, ${ }^{1} \mathrm{~A}$ Rantell, ${ }^{1} \mathrm{~L}$ Cardozo, ${ }^{2} \mathrm{~S}$ Schelfhout, ${ }^{2} \mathrm{H}$ Langerman, ${ }^{2} \mathrm{H}$ Ding, ${ }^{2} \mathrm{~L}$ Hennessy, ${ }^{3} \mathrm{C}$ Atkinson, ${ }^{3} \mathrm{~L}$ Bateman, ${ }^{3} \mathrm{M}$ Silvey, 'SS Birring. ${ }^{1}$ King's College Hospital, London, UK; ${ }^{2}$ Merck and Co., Inc., Kenilworth, USA; ${ }^{3}$ Adelphi Real World, Cheshire, UK

\subsection{6/thorax-2021-BTSabstracts. 168}

Background Stress Urinary Incontinence (SUI) is one of the major physical consequences suffered by individuals with chronic cough (CC). We investigated the prevalence of CC among women who reported having SUI.

Methods Participants completed an online structured quantitative questionnaire in April 2021, to identify adult women with SUI. Demographic characteristics, causes/triggers of urinary incontinence, current or previous CC, cough frequency and duration, COVID-19 infection and its impact on CC were included.

Results A total of 835 adult women reported having SUI, of whom, $153(18.3 \%)$ concomitantly had urgency incontinence, $59(7.1 \%)$ had overflow incontinence, and 28 (3.4\%) had functional incontinence. The mean age was 52.3 years (Range: $21-86)$, the majority $(604(72.3 \%))$ reported cough as a cause of their urinary incontinence, of whom $67.0 \%$ reported suffering incontinence because of cough at least once a week. One hundred and twenty-three (14.7\%) women reported experiencing CC within the last year, and $84(10.1 \%)$ reported still having CC currently. Fifty-seven (6.8\%) women stated their CC had been diagnosed by a physician, and 150 (18.0\%) women reported having suspected or confirmed COVID-19 (with or without CC). Of the 123 women who had CC in the last year, ninety-three $(75.6 \%)$ had CC onset before COVID-19.

Conclusion A majority of women with SUI reported cough as one of the leading triggers of their urinary incontinence. Almost $15 \%$ of the sample reported experiencing CC, but less than half of those had a formal diagnosis from a physician. Most cases of CC were not related to COVID-19. Future studies would be useful to further explore the burden of CC on SUI patients.
Please refer to page A191 for declarations of interest related to this abstract.

\section{P59 BASELINE CHARACTERISTICS AND MEDICAL HISTORY OF PATIENTS WITH REFRACTORY OR UNEXPLAINED CHRONIC COUGH PARTICIPATING IN TWO GLOBAL PHASE 3 CLINICAL TRIALS}

${ }^{1} \mathrm{~L}$ McGarvey, ${ }^{2} \mathrm{SS}$ Birring, ${ }^{3} \mathrm{P}$ Dicpinigaitis, ${ }^{4} \mathrm{~A}$ Morice, ${ }^{5}$ P Pavord, ${ }^{6} \mathrm{JA}$ Smith, ${ }^{7} \mathrm{~B}$ Iskold, ${ }^{7} \mathrm{Q} \mathrm{Li}$, ${ }^{7} \mathrm{~A}$ Martin Nguyen, ${ }^{7} \mathrm{~J}$ Schelfhout, ${ }^{7} \mathrm{~A}$ Tzontcheva, ${ }^{7} \mathrm{C}$ La Rosa, ${ }^{7} \mathrm{D}$ Muccino. ${ }^{1}$ WellcomeWolfson Institute for Experimental Medicine, Queen's University Belfast, Belfast, Ireland; ${ }^{2}$ Centre for Human and Applied Physiological Sciences, School of Basic and Medical Biosciences, Faculty of Life Sciences and Medicine, King's College London, London, UK ${ }^{3}$ Albert Einstein College of Medicine and Montefiore Medical Center, Bronx, USA; ${ }^{4}$ Hull York Medical School, Cottingham, UK; ${ }^{5}$ Oxford NIHR Respiratory BRC, Nuffield Department of Medicine, University of Oxford, Oxford, UK; ${ }^{6}$ Division of Infection, Immunity and Respiratory Medicine, University of Manchester and Manchester University NHS Trust, Manchester, UK; ${ }^{7}$ Merck and Co., Inc., Kenilworth, USA

\subsection{6/thorax-2021-BTSabstracts. 169}

Introduction Previous studies characterizing patients with chronic cough (CC) have typically included patients with CC without a specific focus on patients with guidelines-diagnosed refractory CC (RCC) or unexplained CC (UCC). This analysis assessed the medical history, cough severity, and cough-related quality of life (QOL) at baseline in participants with RCC and UCC enrolled in two global phase 3 trials.

Methods Pooled data from participants enrolled in two phase 3 , randomized, placebo-controlled clinical trials of the P2X3receptor antagonist gefapixant (COUGH-1, NCT03449134; COUGH-2, NCT03449147) were used in this analysis. Participants were adults with cough lasting $\geq 1$ year, a diagnosis of RCC or UCC according to guidelines from the American College of Chest Physicians, and a baseline cough severity score $\geq 40 \mathrm{~mm}$ on a $100-\mathrm{mm}$ visual analog scale. Comorbidities and prior medications were identified from participant medical records. Baseline cough metrics included the Cough Severity Diary (CSD) and Leicester Cough Questionnaire (LCQ). The CSD measures cough severity across 3 domains (frequency, intensity, and disruption), with items measured on an 11-point scale (higher scores indicate greater severity). The LCQ assesses health-related QOL, including physical, psychological, and social domains, with a total score ranging from 3 to 21 (lower scores indicate a more impaired QOL).

Results Of 2044 participants randomized and treated in COUGH-1 or COUGH-2, 41\%, 41\%, and $29 \%$ had prior

\begin{tabular}{lll}
$\begin{array}{l}\text { Abstract P59 Table 1 } \\
\text { COUGH-1 and COUGH-2 }\end{array}$ & Pooled Baseline CSD and LCQ Scores in \\
& Mean (SD) & Median (range) \\
\hline CSD (N=2038) & & \\
Total & $6.0(1.6)$ & $6.1(0.7-10.0)$ \\
Frequency & $6.4(1.5)$ & $6.5(0.8-10.0)$ \\
Intensity & $6.2(1.8)$ & $6.4(0.1-10.0)$ \\
Disruption & $5.3(2.1)$ & $5.4(0.0-10.0)$ \\
LCQ (N=1949) & & \\
Total & $10.4(3.0)$ & $10.3(3.0-20.5)$ \\
Physical & $3.9(1.0)$ & $3.9(1.0-6.8)$ \\
Psychological & $3.2(1.2)$ & $3.1(1.0-7.0)$ \\
Social & $3.2(1.2)$ & $3.3(1.0-7.0)$ \\
\hline CSD, Cough Severity Diary; LCQ, Leicester Cough Questionnaire.
\end{tabular}


diagnoses of asthma, gastroesophageal reflux disease, and rhinitis/upper-airway cough syndrome, respectively; $8 \%$ had prior diagnoses of all 3 conditions. Prior medications were consistent with treatments indicated for these comorbidities or cough and included drugs for obstructive airway diseases (70\%), acid-related disorders (55\%), rhinitis preparations (nasal preparations, 53\%; systemic antihistamines, 35\%), and cough/cold preparations (34\%). Baseline mean total CSD score $(\mathrm{N}=2038)$ was 6.0 and baseline mean total LCQ score $(\mathrm{N}=1949$ ) was 10.4 (table 1).

Conclusions Participants in COUGH-1 and COUGH-2 with RCC or UCC had medical histories consistent with diagnostic and treatment workup of CC according to published guidelines. Participants also reported severe cough with significant cough-related QOL impairment. These data help characterize the profile of patients with RCC or UCC and highlight unmet needs for treatments that can relieve their cough burden.

Please refer to page A191 for declarations of interest related to this abstract.

\section{P60 PATIENT-REPORTED IMPROVEMENTS WITH GEFAPIXANT, A P2X3-RECEPTOR ANTAGONIST, OVER 52 WEEKS IN TWO PHASE 3 CLINICAL TRIALS FOR REFRACTORY OR UNEXPLAINED CHRONIC COUGH}

${ }^{1} \mathrm{SS}$ Birring, ${ }^{2} \mathrm{P}$ Dicpinigaitis, ${ }^{3} \mathrm{~A}$ Morice, ${ }^{4} \mathrm{JA}$ Smith, ${ }^{5} \mathrm{~L}$ McGarvey, ${ }^{6}$ Pavord, ${ }^{7} \mathrm{~A}$ Martin Nguyen, ${ }^{7} \mathrm{~J}$ Schelfhout, ${ }^{7} \mathrm{~A}$ Tzontcheva, ${ }^{7} \mathrm{Q}$ Li, ${ }^{7} \mathrm{C}$ La Rosa, ${ }^{7} \mathrm{D}$ Muccino. ${ }^{7}$ Centre for Human and Applied Physiological Sciences, School of Basic and Medical Biosciences, Faculty of Life Sciences and Medicine, King's College London, London, UK; ${ }^{2}$ Albert Einstein College of Medicine and Montefiore Medical Center, Bronx, USA; ${ }^{3}$ Hull York Medical School, Cottingham, UK; ${ }^{4}$ Division of Infection, Immunity and Respiratory Medicine, University of Manchester and Manchester University NHS Trust, Manchester, UK; ${ }^{5}$ Wellcome-Wolfson Institute for Experimental Medicine, Queen's University Belfast, Belfast, Ireland; ${ }^{6}$ Oxford NIHR Respiratory BRC, Nuffield Department of Medicine, University of Oxford, Oxford, UK; ${ }^{7}$ Merck and Co., Inc., Kenilworth, USA

10.1136/thorax-2021-BTSabstracts. 170

Introduction In two phase 3, randomized, double-blind trials of the P2X3-receptor antagonist gefapixant (COUGH-1 and COUGH-2), participants with refractory or unexplained chronic cough (RCC and UCC) demonstrated significant reductions in 24-hour cough frequency with gefapixant $45 \mathrm{mg}$ twice daily (BID) following 12 (COUGH-1) and 24 (COUGH2) weeks of treatment. Herein, we present a pooled analysis of patient-reported outcomes (PROs) and safety data from an extension of COUGH-1 and COUGH-2 through 52 weeks of treatment.

Methods COUGH-1 and COUGH-2 enrolled participants aged $\geq 18$ years with chronic cough lasting $\geq 1$ year, a diagnosis of RCC or UCC, and a baseline cough severity visual analog scale (VAS) score $\geq 40 \mathrm{~mm}$ on a $100-\mathrm{mm}$ scale. Participants were randomized to receive placebo, gefapixant $15 \mathrm{mg}$ BID, or gefapixant $45 \mathrm{mg}$ BID for 52 weeks. The PROs used to evaluate efficacy included the Leicester Cough Questionnaire (LCQ), cough severity VAS, and Cough Severity Diary (CSD). Logistic-regression models evaluated change from baseline to week 52, where participants were classified as responders as follows: $\geq 1.3$-point increase in LCQ total score, $\geq 30-\mathrm{mm}$ reduction in mean weekly cough severity VAS, and $\geq 1.3$ - and $\geq 2.7$-point reductions in mean weekly CSD total score. Adverse events (AEs) were monitored. Because efficacy was only demonstrated with gefapixant $45 \mathrm{mg}$ BID, only the

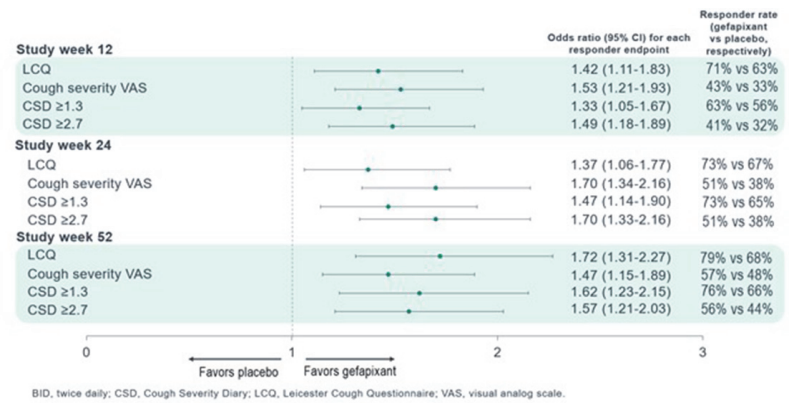

Abstract P60 Figure 1 Patient-reported outcomes for gefapixant 45 mg BID vs placebo at weeks 12, 24, and 52 pooled across COUGH-1 and COUGH-2

placebo and gefapixant $45 \mathrm{mg}$ BID cohorts are presented in this report.

Results There were 2044 participants included in the pooled data set. Across all PROs and time points, gefapixant $45 \mathrm{mg}$ BID was consistently favored over placebo (figure 1). Frequently reported AEs were taste related. Discontinuations due to taste-related AEs were $14 \%$ vs $<1 \%$ in the gefapixant 45 mg BID vs placebo cohorts, respectively. Serious AEs occurred in $6 \%$ of participants in each cohort.

Conclusions Treatment for 52 weeks with gefapixant $45 \mathrm{mg}$ BID resulted in clinically meaningful patient-reported efficacy relative to placebo across all PROs. Taste-related AEs led to discontinuations in a small proportion of participants who received gefapixant, and the occurrence of serious AEs with gefapixant was similar to that of placebo. These data support the long-term, patient-relevant efficacy of gefapixant $45 \mathrm{mg}$ BID for treatment of RCC or UCC.

\section{P61 POOLED ANALYSIS OF OBJECTIVE COUGH FREQUENCY IN PARTICIPANTS WITH CHRONIC COUGH TREATED WITH GEFAPIXANT IN TWO PHASE 3 CLINICAL TRIALS (COUGH-1 AND COUGH-2)}

${ }^{1} \mathrm{JA}$ Smith, ${ }^{2} \mathrm{~A}$ Morice, ${ }^{3} \mathrm{~L}$ McGarvey, ${ }^{4} \mathrm{P}$ Pavord, ${ }^{5} \mathrm{SS}$ Birring, ${ }^{6} \mathrm{P}$ Dicpinigaitis, ${ }^{7} \mathrm{~B}$ Iskold, ${ }^{7} \mathrm{Q} \mathrm{Li}$, ${ }^{7} \mathrm{~A}$ Tzontcheva, ${ }^{7} \mathrm{C}$ La Rosa, ${ }^{7} \mathrm{D}$ Muccino. ${ }^{1}$ Division of Infection, Immunity and Respiratory Medicine, University of Manchester and Manchester University NHS Trust, Manchester, UKi ${ }^{2}$ Hull York Medical School, Cottingham, UK; ${ }^{3}$ Wellcome-Wolfson Institute for Experimental Medicine, Queen's University Belfast, Belfast, Ireland; ${ }^{4}$ Oxford NIHR Respiratory BRC, Nuffield Department of Medicine, University of Oxford, Oxford, UK; ${ }^{5}$ Centre for Human and Applied Physiological Sciences, School of Basic and Medical Biosciences, Faculty of Life Sciences and Medicine, King's College London, London, UK; ${ }^{6}$ Albert Einstein College of Medicine and Montefiore Medical Center, Bronx, USA; ${ }^{7}$ Merck and Co., Inc., Kenilworth, USA

\subsection{6/thorax-2021-BTSabstracts. 171}

Introduction Chronic cough (CC) is a relatively common condition often associated with comorbidities such as asthma, gastroesophageal reflux disease, or upper-airway cough syndrome. A subset of patients experience CC that does not resolve after treatment of cough-associated conditions (refractory CC [RCC]) or for which there is no known cause of CC despite clinical evaluation according to published guidelines (unexplained CC [UCC]). However, no treatments are currently approved for RCC or UCC. In two large phase 3 trials (COUGH-1, NCT03449134; COUGH-2, NCT03449147), the P2X3-receptor antagonist gefapixant demonstrated significant reductions in the primary endpoint (24-hour cough frequency) in participants with RCC or UCC at a dosage of $45 \mathrm{mg}$ twice daily (BID) vs 